\title{
A comparison of the amino acid profiles of the pea aphid, Acyrthosiphon pisum, and the social aphid species, Pemphigus spyrothecae (Hemiptera: Aphididae)
}

\author{
NATHAN PIKE
}

Department of Zoology, University of Oxford, South Parks Road, Oxford OX1 3PS, UK; e-mail: nathan.pike.1998@pem.cam.ac.uk

Key words. Hemiptera, Aphididae, Pemphigus spyrothecae, Acyrthosiphon pisum, artificial diet, soldier aphid, plant gall, amino acid profile, high performance liquid chromatography

\begin{abstract}
The relative proportions of free amino acids as well as the amino acid compositions of hydrolysed unprecipitated peptides and hydrolysed whole carcasses were quantified for two aphid species: the gall-dwelling social aphid Pemphigus spyrothecae and the pea aphid Acyrthosiphon pisum. The whole-tissue amino acid profiles of the two taxonomically distant species had a surprisingly high level of correspondence. In contrast, when comparing the A. pisum profiles obtained in the current study to those obtained in an earlier study, major differences were identified. It is concluded that there are good prospects for developing an artificial diet for $P$. spyrothecae. There may also exist considerable scope for tailoring the existing diets of A. pisum to suit specialised populations which develop poorly on the standard diet. The amino acid profile of $P$. spyrothecae is the first such profile that has been reported for a gall-forming aphid.
\end{abstract}

\section{INTRODUCTION}

Artificial diets have been developed for a range of economically significant sap-feeding insects including mirids, lygaeids, coccids (Auclair, 1969), cicadellids (Hou \& Lin, 1979) and, of course, aphids such as the pea aphid, Acyrthosiphon pisum (Febvay et al., 1988) and the peach-potato aphid, Myzus persicae (Mittler \& Kleinjan, 1970). These artificial diets can be strictly regulated and serve the important function of enabling the insect study organisms to be reared away from their natural host plants in controlled environments. Biologists have relied on artificial diets to conduct groundbreaking research that has elucidated insect life histories and provided valuable information on which chemical and biological agents can be used to effectively control populations of pest insects. Indeed, many studies would have been impossible without the artificial diets that have enabled the establishment of constant laboratory cultures of insects which have intermittent or deciduous host plants that would otherwise impose a seasonal occurrence/semi-ennial life cycle.

An artificial diet has been used very effectively in a series of studies that elucidates the mechanisms underlying the social life-history strategies of an Asian species of galling aphid, Tuberaphis styraci (Shibao et al., 2002, 2003, 2004). In natural conditions, this species has a holocyclic and biennial life cycle of a complexity that precludes laboratory culture. In identifying and establishing their remarkable study system, Shibao et al. (2002) used a process of elimination: they trialed 16 socialaphid species on a single diet developed for the distantlyrelated $A$. pisum and found that $T$. styraci was the only species to develop satisfactorily. The research reported in the current paper represents the founding step in an alternative approach through which an artificial diet is tailored for a particular species. The species of interest is Pemphigus spyrothecae, a natively European aphid which forms galls on poplar, Populus nigra, and which possesses a number of social traits including aggressive, morphologically distinct soldiers (Pike et al., 2007). As the diet of $A$. pisum was developed by emulating the amino acid profile of hydrolysed whole tissue of the organism (Febvay et al., 1988; Sasaki et al., 1991), the aim of the current work was to profile the amino acid composition of $P$. spyrothecae. The amino acid profile of $A$. pisum was also assessed using the same apparatus such that the profiles of the two species could be meaningfully compared.

\section{MATERIAL AND METHODS}

$P$. spyrothecae was sampled directly from natural populations found on Lombardy poplar trees in Oxford whereas A. pisum samples were obtained from laboratory cultures reared on broad beans (Vicia faba). An Applied Biosystems Model 420A PTC derivatiser and an Applied Biosystems Model 130A PTC Amino Acid Analyzer were used to quantify amino acid composition via high performance liquid chromatography. Fourth-instar virginoparous nymphs of both $P$. spyrothecae and $A$. pisum (three of each species) were used to produce samples of the following types: (1) free amino acids, (2) non-precipitated peptides, and (3) hydrolysed whole-aphid tissue. Preparing samples of type (1) involved crushing a whole individual aphid in $5 \%$ trichloracetic acid, addition of 2 nmols norvaline as an internal standard, centrifugation, and washing the supernatant with chloroform twice. Half of this aqueous supernatant was retained to allow the composition of free amino acids to be analysed. The other half of the supernatant was used to create samples of type (2) by drying under vacuum and hydrolysing for 24 $\mathrm{h}$ at $110^{\circ} \mathrm{C}$ in a vial containing $5.7 \mathrm{~N} \mathrm{HCl}$ and a small amount of phenol. The resulting sample was dissolved in distilled water before amino acid analysis. The three replicate samples of type (3) were obtained by directly hydrolysing the whole tissue of a fourth-instar individual using the vapour-phase hydrolysis protocol just described. Phenylthiocarbamyl (PTC) amino acid derivatives were obtained by reacting the free or hydrolysed amino acids with phenylisothiocyanate under basic conditions. A methanol solution of these derivatives was then transferred to a narrow-bore high performance liquid chromatography system which used a reverse phase C18 silica separation column. Elution was conducted over $25 \mathrm{~min}$ in combined buffer solutions of pH $5.5120 \mathrm{mM}$ sodium acetate and 70\% acetonitrile. The acetonitrile solution initially comprised $7 \%$ of the buffer, increasing to $60 \%$ concentration at the end of the gradient. The chromo- 
$\mathrm{T}_{\mathrm{ABLE}}$ 1. The amino acid compositions (in molar percentages, average values \pm SE calculated across three individuals for each species) of fourth-instar nymphs of $P$. spyrothecae and A. pisum. *Because asparagine is deamidated to aspartic acid during hydrolysis, these values represent Asp + Asn. ${ }^{* *}$ Similarly, these values represent Glu + Gln. Dashes indicate that tryptophan is destroyed by the acid hydrolysis used in preparing peptidic and whole-tissue samples.

\begin{tabular}{|c|c|c|c|c|c|c|}
\hline \multirow{2}{*}{ Amino acid } & \multicolumn{3}{|c|}{ Pemphigus spyrothecae } & \multicolumn{3}{|c|}{ Acyrthosiphon pisum } \\
\hline & (1) Free & (2) Peptidic & (3) Whole tissue & (1) Free & (2) Peptidic & (3) Whole tissue \\
\hline Asp & $6.4 \pm 0.1$ & $6.7 \pm 0.1^{*}$ & $6.0 \pm 0.2^{*}$ & $2.4 \pm 0.2$ & $14.1 \pm 0.8^{*}$ & $7.7 \pm 0.4 *$ \\
\hline Asn & $14.6 \pm 0.6$ & & & $3.8 \pm 0.1$ & & \\
\hline Thr & $1.4 \pm 0.1$ & $3.9 \pm 0.2$ & $6.1 \pm 0.2$ & $5.1 \pm 0.2$ & $1.4 \pm 0.1$ & $5.4 \pm 0.3$ \\
\hline Ser & $1.8 \pm 0.1$ & $6.8 \pm 0.2$ & $7.6 \pm 0.2$ & $5.7 \pm 0.2$ & $3.0 \pm 0.1$ & $7.4 \pm 0.3$ \\
\hline Glu & $10.7 \pm 0.3$ & $17.7 \pm 0.9 * *$ & $5.6 \pm 0.1 * *$ & $10.1 \pm 0.6$ & $34.5 \pm 1.7 * *$ & $7.5 \pm 0.1^{* *}$ \\
\hline Gln & $8.2 \pm 0.3$ & & & $0.7 \pm 0.1$ & & \\
\hline Pro & $5.2 \pm 0.1$ & $5.7 \pm 0.4$ & $7.4 \pm 0.3$ & $7.2 \pm 0.4$ & $4.1 \pm 0.2$ & $7.1 \pm 0.2$ \\
\hline Gly & $2.7 \pm 0.2$ & $9.9 \pm 0.1$ & $10.9 \pm 0.4$ & $6.7 \pm 0.5$ & $5.9 \pm 0.1$ & $10.6 \pm 0.3$ \\
\hline Ala & $18.5 \pm 0.3$ & $21.0 \pm 0.7$ & $11.5 \pm 0.4$ & $22.2 \pm 1.3$ & $12.3 \pm 0.1$ & $12.7 \pm 0.4$ \\
\hline Val & $0.6 \pm 0.1$ & $0.9 \pm 0.1$ & $2.3 \pm 0.1$ & $0.9 \pm 0.1$ & $1.7 \pm 0.1$ & $2.7 \pm 0.1$ \\
\hline Met & $2.7 \pm 0.1$ & $4.0 \pm 0.1$ & $8.1 \pm 0.4$ & $4.9 \pm 0.1$ & $2.1 \pm 0.1$ & $7.3 \pm 0.1$ \\
\hline Ile & $1.2 \pm 0.1$ & $2.4 \pm 0.2$ & $7.4 \pm 0.5$ & $2.7 \pm 0.1$ & $1.2 \pm 0.1$ & $6.1 \pm 0.4$ \\
\hline Leu & $1.5 \pm 0.1$ & $3.5 \pm 0.2$ & $9.8 \pm 0.4$ & $3.6 \pm 0.1$ & $1.6 \pm 0.1$ & $8.6 \pm 0.6$ \\
\hline Tyr & $5.8 \pm 0.1$ & $6.8 \pm 0.3$ & $4.2 \pm 0.3$ & $10.9 \pm 0.1$ & $4.0 \pm 0.1$ & $5.4 \pm 0.1$ \\
\hline Phe & $1.4 \pm 0.1$ & $1.7 \pm 0.1$ & $4.1 \pm 0.1$ & $2.3 \pm 0.1$ & $1.0 \pm 0.1$ & $3.7 \pm 0.2$ \\
\hline Lys & $9.5 \pm 0.6$ & $5.4 \pm 0.1$ & $3.3 \pm 0.2$ & $4.0 \pm 0.1$ & $7.5 \pm 0.2$ & $3.6 \pm 0.1$ \\
\hline His & $3.1 \pm 0.2$ & 0 & 0 & $2.2 \pm 0.1$ & $2.0 \pm 0.1$ & 0 \\
\hline Arg & $4.7 \pm 0.3$ & $3.6 \pm 0.1$ & $5.7 \pm 0.1$ & $4.6 \pm 0.3$ & $3.4 \pm 0.2$ & $6.2 \pm 0.2$ \\
\hline Trp & $0.1 \pm 0.1$ & - & - & 0 & - & - \\
\hline
\end{tabular}

phore of the derivatives was detected at $254 \mathrm{~nm}$. Chromatic peak areas were quantified using Dionex Chromeleon software and molar percentages were calculated for each amino acid identified. Mean amino acid compositions were compared across the two species via analysis of variance.

\section{RESULTS}

The amino acid compositions of $P$. spyrothecae and $A$. pisum are set out in Table 1. Notable differences in free amino acid compositions of $P$. spyrotheacae vs $A$. pisum were seen in asparagine $\left(14.6 \%\right.$ vs $\left.3.8 \%, F_{1,4}=506.72, p<0.001\right)$, glutamine $\left(8.2 \%\right.$ vs $\left.0.7 \%, F_{1,4}=488.89, p<0.001\right)$, lysine $(9.5 \%$ vs $4.0 \%$, $\left.F_{1,4}=80.39, p=0.001\right)$, and tyrosine $\left(5.8 \%\right.$ vs $10.9 \%, F_{1,4}=$ $1117.10, p<0.001)$. The key between-species differences found in the assays of unprecipitated peptides were in glutamic acid + glutamine $\left(17.7 \%\right.$ vs $\left.34.5 \%, F_{1,4}=76.72, p=0.001\right)$, alanine $\left(21.0 \%\right.$ vs $\left.12.3 \%, F_{1,4}=162.42, p<0.001\right)$, and aspartic acid $\left(6.7 \%\right.$ vs $\left.14.1 \%, F_{1,4}=77.33, p=0.001\right)$. Between-species differences in the amino acid composition of hydrolysed wholetissue samples were relatively small, the two greatest differences being seen in glutamic acid + glutamine $(5.6 \%$ vs $7.5 \%$, $\left.F_{1,4}=330.13, p<0.001\right)$ and aspartic acid $\left(6.0 \%\right.$ vs $7.7 \%, F_{1,4}=$ $15.17, p=0.02$ ).

The results reported here for $A$. pisum feeding on broad beans were compared to those of Febvay et al. (1988). Notably, in comparing the whole-tissue results, the level of glutamic acid + glutamine was significantly lower in the current study $(8 \%$ vs $16 \%$ ). Lower levels of glutamic acid + glutamine (35\% vs $54 \%$ ) were also found in the unprecipitated peptidic samples. At the level of free amino acids, the percentage composition of glutamine alone was found to be much lower in the current study's assays than in those of Febvay et al. (1\% vs 12\%). The hydrolysed whole-tissue assays of A. pisum reported here also differed from those of Febvay et al. in being higher in methionine $(7 \%$ vs $1 \%)$ and lower in valine ( $3 \%$ vs $8 \%$ ). The levels of alanine were also higher in the current study for all sample types $(13 \%$ vs $8 \%$ for hydrolysed whole tissue, $12 \%$ vs $1 \%$ for hydrolysed unprecipitated peptides, and $22 \%$ vs $9.5 \%$ for free amino acids).
Finally, it should be noted that the level of free asparagine was much lower in the current study than in that of Febvay et al. $(3.8 \%$ vs $21.4 \%)$.

\section{DISCUSSION}

The similarity in the hydrolysed whole-tissue amino acid compositions of the two species is remarkable, not least because the species have markedly different host plants (a tree for $P$. spyrothecae and legumes for $A$. pisum) and are taxonomically distant. [Adhering to the taxonomy of Remaudière \& Remaudière (1997), $P$. spyrothecae is a pemphigine while $A$. pisum is an aphidine.] If, as for A. pisum (Febvay et al., 1988), the artificial diet of $P$. spyrothecae can be optimised by mimicking the whole-tissue amino acid composition, this similarity may well permit both species to be raised on the same or very similar artificial diets.

The large differences between the pea aphid samples of the two studies is somewhat unexpected and cannot be attributed directly to differences in either the host plant or the developmental stage of the aphid tested: in both studies, assays were performed on fourth-instar individuals which had been reared on seedlings of $V$. faba. The "biotype" used by Febvay et al. (1988) was sourced in the area of Lusignan, France from an unrecorded host plant while the clone used in this study was originally collected in the area of Ascot, UK from Vicia sp. Populations of $A$. pisum do evolve genetically-based preferences for host plant species (Ferrari et al., 2006) and these specialisations may well underlie differences in amino-acid composition such as those that the current study has documented. Of course, the implication of such differences for insect husbandry through artificial diets is that quite major reformulations might produce significant benefits even within a single species.

There are grounds to expect less intra-specific variation in amino acid composition in $P$. spyrothecae than has been recorded for A. pisum. P. spyrothecae is monophagus and therefore may be evolutionarily constrained to a narrow range of diets and to having a relatively stable amino-acid profile. The unstable profile of $A$. pisum might equally be viewed as a conse- 
quence of the fact that, generally speaking, the species is adapted for life on a large variety of host plants. [Note that this broad host range is at least partly due to the microbial symbionts of A. pisum which can compensate for nutritional deficiencies (Sasaki et al., 1991; Tsuchida et al., 2004)]. The outlook for the development of a universal artifical diet for use in laboratory cultures of $P$. spyrothecae is thus promising.

ACKNOWLEDGEMENTS. A. Willis of the Department of Biochemistry, University of Oxford provided advice and invaluable technical assistance in the amino acid analyses. J. Ferrari of the University of York provided stocks of A. pisum. A Royal Society grant provided financial support for the study.

\section{REFERENCES}

AuClair J.L. 1969: Nutrition of plant-sucking insects on chemically defined diets. Entomol. Exp. Appl. 12: 623-641.

Febvay G., Delobel B. \& Rahbé Y. 1988: Influence of the amino acid balance on the improvement of an artificial diet for a biotype of Acyrthosiphon pisum (Homoptera: Aphididae). Can. J. Zool. 66: 2449-2453.

Ferrari J., Godfray H.C.J., Faulconbridge A.S., Prior K. \& VIA S. 2006: Population differentialation and genetic variation in host choice among pea aphids from eight host plant genera. Evolution 60: 1574-1584.
Hou R.F. \& Lin L.C. 1979: Artificial rearing of the rice green leafhopper, Nephotettix cincticeps, on a holidic diet. Entomol. Exp. Appl. 25: 158-164.

Mittler T. \& Kleinjan J. 1970: Effect of artificial diet composition on wing-production by the aphid Myzus persicae. $J$. Insect Physiol. 16: 833-850.

Pike N., Whitfield J. \& Foster W. 2007: Ecological correlates of sociality in Pemphigus aphids, with a partial phylogeny of the genus. BMC Evol. Biol. 7: 185.

Remaudière G. \& Remaudière M. 1997: Catalogue des Aphides $d u$ Monde. INRA, Paris, $467 \mathrm{pp}$.

Sasaki T., Hayashi H. \& IshiKawa H. 1991: Growth and reproduction of the symbiotic and aposymbiotic pea aphids, Acyrthosiphon pisum, maintained on artificial diets. J. Insect Physiol. 37: 749-756.

Shibao H., Kutsukake M., Lee J.-M. \& Fukatsu T. 2002: Maintenance of soldier-producing aphids on an artificial diet. $J$. Insect Physiol. 48: 495-505.

Shibao H., Lee J. Kutsukake M. \& Fukatsu T. 2003: Aphid soldier differentiation: density acts on both embryos and newborn nymphs. Naturwissenschaften 90: 501-504.

Shibao H., Kutsukake M. \& Fukatsu T. 2004: Densitydependent induction and suppression of soldier differentiation in an aphid social system. J. Insect Physiol. 50: 995-1000.

Tsuchida T., Koga R. \& Fukatsu T. 2004: Host plant specialization governed by a facultative symbiont. Science 303: 1989.

Received June 24, 2009; revised and accepted March 5, 2010 